Univerzitet u Beogradu
Poljoprivredni fakultet
Institut za poljoprivrednu tehniku
Naučni časopis
POLJOPRIVREDNA TEHNIKA
Godina XLV
Broj 1, 2020.
Strane: $38-50$

\title{
EFFECT OF MOISTURE CONTENT AND LOADING POSITIONS ON THE MECHANICAL PROPERTIES OF NEW RICE FOR AFRICA (NERICA)
}

\author{
Eze Paul Chukwuka*1, Oluka Sylvester Ikechukwu ${ }^{1}$, Eze Chikaodili Nkechi ${ }^{1}$ \\ ${ }^{1}$ Agricultural and Bioresource Engineering, \\ Enugu State University of Science and Technology, \\ Enugu, Nigeria.
}

\begin{abstract}
The mechanical properties of New Rice for Africa, (NERICA) were determined on different moisture content levels of $12.5,17$ and $21.5 \%(\mathrm{db})$, and loading positions of major, minor, and intermediate diameters using the Instron Universal Testing Machine (UTM). The mechanical properties studied were the maximum load at rupture, compressive extension at maximum load, load at bioyield point, toughness, stiffness, compressive strength, energy at maximum load, and the maximum slope. Results obtained indicated that, the loading pattern and moisture content affected the mechanical properties determined $(\mathrm{p}<0.05)$. Results showed that, maximum load at rupture, compressive extension, load at bio yield point, ranged from 363.73 to $50.92 \mathrm{~N}$; 0.50 to $2.79 \mathrm{~mm}$; and 11.58 to $6.78 \mathrm{~N} / \mathrm{mm}^{2}$; respectively for NERICA Raw-Paddy major diameter loading; and from 18.46 to $5.44 \mathrm{~N} ; 0.92$ to $2.52 \mathrm{~mm} ; 9.01$ to $4.08 \mathrm{~N} / \mathrm{mm}^{2}$ respectively for NERICA Raw-Paddy minor diameter loading. For the NERICA Parboiled-Milled, results at both major diameter and minor diameter loading positions ranged as follows; maximum load at rupture (156.08 to $44.94 \mathrm{~N} ; 94.90$ to $22.05 \mathrm{~N})$; compressive extension ( 0.59 to $1.61 \mathrm{~mm}$; 0.46 to $2.67 \mathrm{~mm}$ ). Regression analysis were carried out on the mechanical properties with moisture content, and there was positive correlation between the parameters. There were significant effects of moisture content ( $\mathrm{p}$ $<0.05)$ on all parameters studied.
\end{abstract}

Keywords: Moisture content, Mechanical properties, loading position, NERICA.

\footnotetext{
${ }^{*}$ Corresponding Author. E-mail: paul.eze@esut.edu.ng
} 


\section{INTRODUCTION}

The word NERICA means "New Rice for Africa" and this is used to represent inborn products developed from the effective crossing of two rice cultivars; the African rice, which is O.glaberrima steud, and the Asian rice, which is O. Sativa L., in order to generate offspring which brings together the first-class qualities of the two parentages, [1]. These involves high-level vintages from the Asian specie and the capability from the African specie to grow vigorously in an unpleasant and difficult environment. NERICA was developed by normal cross breeding and on that ground, they are not hereditarily improved rice, [1]. NERICA varieties are new class of highland varieties of rice which ideally accommodate to the rain-fed highland environment in the Sub-Saharan Africa (SSA), where poor farmers do not have access to irrigation, chemical fertilizers or pesticides. Reports from the Rice centre showed that, NERICA varieties also react positively than the local varieties to greater inputs. The NERICA varieties gives hope to multitudes of poverty-stricken rice farmers, and for numerous other farmers that works hard in a very bad and dirty conditions, spending majority of their little income on rice, [1]. Rice generally is a very important convenience food in Nigeria and much of SubSahara Africa, and currently Nigeria is regarded as the largest rice producing country in Africa. This is as a result of steadily increasing incomes from rice and as well as continued population growth, [2]. However, the increasing demand for rice, both in quantity and quality, far overshadowed local production. Thus, the need to increase production and improve the locally produced rice to make it more competitive with imported rice led to the discovery of NERICA.

Moreover, intensifying cost-effective significance of agricultural food resources, in conjunction with the complications of contemporary technology for their processing, handling, storage and preservation, quality assessment, distribution, marketing and consumption, requires wide-ranging knowledge on engineering properties of these agricultural materials pertinent to their processing, handling, storage, and preservation. Information on the mechanical properties of agricultural materials are considered necessary in the designing and modification of machines and some parameters utilized during processing, and storage of agricultural products as well as in converting them into food, feed and fibre, [3].

These properties effect the designing and assessment of rice processing which includes; drying, shelling, blanching and shining as well as sorting equipment, storing and grain handling equipment, [4]. So, to obtain better quality-milled NERICA, packaging and further processing, the knowledge of mechanical properties of the grain are essential for modeling of dynamic abrasion in rice molding operations as well as for designing of appropriate polishing systems, [5]. Mechanical property of food is described as the discipline which investigates the agricultural product force-deformation and flow. Information from analysis of mechanical properties is needed in agricultural material evaluation quality, computation of engineering data, and process design.

The knowledge on force-deformation characteristics and behavior are very necessary to decide or establish actual parameters needed for process design, estimating other properties, characterizing foods, and quality determination and energy needed during processing of food. 
Models obtained from the mechanical property parameters calculations from the measured experiments could be highly effective in the design of food process when applied concurrently through energy, force, and load proportions. There are several researches conducted on mechanical properties involved with rice and other agricultural products such as $[6,7,8,9$, and 10], and others. Therefore, this study is aimed at determining the effect of moisture content and loading patterns on the mechanical properties of NERICA varieties relevant to their processing and handling.

\section{MATERIALS AND METHOD}

Source of Material and Sample Preparation

The research materials used in this study include five varieties of NERICA, and they are; FAROs 44, 52, 57, 60, and 61. These varieties of NERICA were collected from the Ebonyi State Agricultural Development Programme (EBADEP), Abakaliki, at storage moisture content of $12.5 \%(\mathrm{db})$. Some of the paddy from each variety were parboiled and dehulled using a rice dehulling machine to obtain parboiled-milled samples of the NERICA varieties thereby having ten samples of NERICA; viz: FAROs 44, 52, 57, 60, and 61 Raw-Paddy and FAROs 44, 52, 57, 60, and 61 Parboiled-Milled. The methods used in the parboiling and dehulling were in line with the rice parboiling and dehulling standard, and these processes are cleaning, soaking, steaming, drying and milling, [11]. The sample varieties were further hydrated to acquire more three different moisture content levels at which the tests were carried out. A total of 1000 grains were used for the experiments.

\section{Methods}

\section{Determination of weight of the grains}

The measurement of the weight of the sample grains were carried out using the Mettler Toledo analytical weighing machine of model XP 204 and $0.0001 \mathrm{~g}$ sensitivity. Measurements were replicated 20 times. Also, a thousand-grain weight of the sample seeds were carried out by picking 100 seed samples and weighing them using the analytical weighing machine, then multiplying them by 10 in order to get the 1000 grain mass of the sample materials. This was done using reported method by [12].

Determination of Moisture Content

The oven was used to dry the hydrated samples and the moisture content analytical machine was used in measuring the moisture content of the sample materials at room temperature, and these were further evaluated by using equation 1 as reported by [3].

$$
M c=\frac{W w-D w}{D w} \times 100 \%
$$

where; $\boldsymbol{M} \boldsymbol{c}=$ moisture content, $\% ; \boldsymbol{W} \boldsymbol{w}=$ wet samples; and $\boldsymbol{D} \boldsymbol{w}=$ dried samples. 
Determination of the Mechanical Properties:

Force - deformation measurements of NERICA varieties were conducted at varying moisture contents using an Instron Universal Testing Machine (UTM) of model 3340 series single column, USA, controlled by a fully packed Dell computer with window 8 version and were installed with the latest Instron Blue Hill 3 Software. Three loading orientations namely; major, minor and intermediate diameters were used during the tests.

The NERICA samples were deformed at the crosshead speed of $10 \mathrm{~mm}$ per min. As deformation of the NERICA samples progresses and advances, a load - deformation curve was automatically plotted in relation towards the response of the NERICA sample to compressions. Randomly picked NERICA samples were tested at each sample's moisture content and loading pattern and the process was repeated three times and the obtained results were analyzed for; Maximum load (rupture point) (N); Compressive extension at maximum load (deformation) (mm); Load at bioyield point (N); Energy at maximum load (J); Maximum slope or (Automatic Young's modulus of deformability) $(\mathrm{mm} / \mathrm{mm})$. Toughness, which is the capability of an agricultural material to take up energy and plastically deform without rupturing, was computed using the equation 2 as reported by [13];

$$
\text { Toughness }=\frac{\text { Rupture Energy }}{\text { Volume of Material }}\left(\mathrm{N} / \mathrm{mm}^{2}\right)
$$

Stiffness modulus was also calculated from the equation 3 according to [7];

$$
\text { Stiffness modulus }=\frac{\text { Max breaking force }}{\text { Max deformation at breaking }}\left(\frac{N}{m m}\right)
$$

\section{RESULTS AND DISCUSSION}

The results of the mechanical properties of the five varieties of NERICA (FAROs $44,52,57,60$, and 61) are presented in Tables 1 to 6 . The moisture contents of $12.5 \%$, $17 \%$ and $21.5 \%(\mathrm{db})$ were obtained and used to determine the mechanical properties under major diameter, minor diameter and intermediate diameter loading positions.

Table 1. Mechanical Properties of NERICA varieties (Raw-Paddy) at $12.5 \%$ (d.b)

\begin{tabular}{|c|c|c|c|c|}
\hline \multirow[b]{2}{*}{$\begin{array}{l}\text { Mechanical } \\
\text { Properties }\end{array}$} & \multirow[b]{2}{*}{ NERICA Variety } & \multicolumn{3}{|c|}{ Loading Positions } \\
\hline & & Major Diameter & Minor Diameter & $\begin{array}{c}\text { Intermediate } \\
\text { Diameter }\end{array}$ \\
\hline \multirow{6}{*}{$\begin{array}{l}\text { Maximum Load at } \\
\text { Rupture [N] }\end{array}$} & FARO 44 & $233.44(1.03)$ & $9.06(0.09)$ & $80.84(1.56)$ \\
\hline & FARO 52 & $336.90(0.42)$ & $10.36(1.13)$ & $115.75(0.97)$ \\
\hline & FARO 57 & 270.87 (2.09) & $18.46(0.07)$ & $96.44(2.03)$ \\
\hline & FARO 60 & $363.73(1.87)$ & $12.31(1.06)$ & $125.35(0.11)$ \\
\hline & FARO 61 & $279.17(0.67)$ & $18.01(0.43)$ & $99.06(0.57)$ \\
\hline & AVERAGE & $296.82(1.22)$ & $13.64(0.56)$ & $103.49(1.05)$ \\
\hline \multirow{6}{*}{$\begin{array}{c}\text { Compressive } \\
\text { Extension at } \\
\text { Maximum Load }[\mathrm{mm}\end{array}$} & FARO 44 & $1.60(0.23)$ & $1.88(0.98)$ & $1.71(0.09)$ \\
\hline & FARO 52 & $1.53(0.18)$ & $1.51(1.06)$ & $1.48(0.23)$ \\
\hline & FARO 57 & $1.76(1.53)$ & $2.02(1.83)$ & $1.82(1.07)$ \\
\hline & FARO 60 & $1.40(2.09)$ & $1.24(0.45)$ & $1.29(0.44)$ \\
\hline & FARO 61 & $0.50(0.18)$ & $0.92(1.09)$ & $0.68(0.99)$ \\
\hline & AVERAGE & $1.36(0.84)$ & $1.52(1.08)$ & $1.41(0.56)$ \\
\hline
\end{tabular}
under different loading positions 


\begin{tabular}{|c|c|c|c|c|}
\hline \multirow{6}{*}{$\begin{array}{c}\text { Load at Bio yield } \\
\text { Point }[N]\end{array}$} & FARO 44 & $200.25(0.65)$ & $6.50(0.87)$ & $67.88(1.66)$ \\
\hline & FARO 52 & $250.19(1.08)$ & $7.92(1.06)$ & $91.45(2.02)$ \\
\hline & FARO 57 & $179.89(2.02)$ & $8.98(0.98)$ & $63.89(0.56)$ \\
\hline & FARO 60 & $321.56(0.34)$ & $7.87(1.23)$ & $88.76(0.88)$ \\
\hline & FARO 61 & $220.76(0.08)$ & $6.78(0.89)$ & $63.45(1.44)$ \\
\hline & AVERAGE & $234.53(0.83)$ & $7.61(1.01)$ & $75.09(1.31)$ \\
\hline \multirow{6}{*}{$\begin{array}{l}\text { Toughness } \\
\left(\mathrm{N} / \mathrm{mm}^{2}\right)\end{array}$} & FARO 44 & $11.58(0.98)$ & $9.01(2.09)$ & $10.12(1.11)$ \\
\hline & FARO 52 & $10.96(0.56)$ & 8.98 (1.07) & $9.45(0.97)$ \\
\hline & FARO 57 & $8.89(1.34)$ & $6.06(0.56)$ & $7.32(1.06)$ \\
\hline & FARO 60 & $9.98(0.94)$ & $6.72(0.74)$ & $7.98(0.99)$ \\
\hline & FARO 61 & $8.67(2.07)$ & $7.08(0.31)$ & $7.23(0.78)$ \\
\hline & AVERAGE & $10.02(1.18)$ & $7.57(0.95)$ & $8.42(0.98)$ \\
\hline \multirow{6}{*}{$\begin{array}{l}\text { Stiffness } \\
(\mathrm{N} / \mathrm{mm})\end{array}$} & FARO 44 & $36.89(1.05)$ & $34.70(1.32)$ & $35.12(0.77)$ \\
\hline & FARO 52 & $39.56(0.97)$ & $45.78(2.09)$ & $43.42(2.07)$ \\
\hline & FARO 57 & $48.98(1.04)$ & $46.98(0.93)$ & $47.27(0.54)$ \\
\hline & FARO 60 & $38.76(2.05)$ & $32.76(1.04)$ & 36.34 (1.97) \\
\hline & FARO 61 & $43.72(0.57)$ & $40.07(0.39)$ & $42.97(0.45)$ \\
\hline & AVERAGE & $41.58(1.14)$ & $40.06(1.15)$ & $41.02(1.16)$ \\
\hline \multirow{6}{*}{$\begin{array}{c}\text { Compressive } \\
\text { Strength } \\
\left(\mathrm{N} / \mathrm{mm}^{2}\right)\end{array}$} & FARO 44 & $6.91(0.54)$ & $5.21(0.56)$ & $6.01(0.62)$ \\
\hline & FARO 52 & $5.78(0.88)$ & $5.04(1.67)$ & $5.29(2.03)$ \\
\hline & FARO 57 & $5.21(0.78)$ & $4.32(0.96)$ & $4.61(1.00)$ \\
\hline & FARO 60 & $6.22(0.65)$ & $4.89(2.03)$ & $5.34(0.31)$ \\
\hline & FARO 61 & $5.38(0.42)$ & $5.09(0.57)$ & $5.11(0.46)$ \\
\hline & AVERAGE & $5.90(0.65)$ & $4.91(1.16)$ & $5.27(0.88)$ \\
\hline \multirow{6}{*}{$\begin{array}{c}\text { Energy at Maximum } \\
\text { Load [J] }\end{array}$} & FARO 44 & $0.06(0.02)$ & $0.05(0.01)$ & $0.05(0.02)$ \\
\hline & FARO 52 & $0.11(0.08)$ & $0.03(0.10)$ & $0.09(0.10)$ \\
\hline & FARO 57 & $0.05(0.04)$ & $0.04(0.09)$ & $0.05(0.01)$ \\
\hline & FARO 60 & $0.14(0.10)$ & $0.02(0.01)$ & $0.07(0.02)$ \\
\hline & FARO 61 & $0.08(0.03)$ & $0.04(0.02)$ & $0.06(0.03)$ \\
\hline & AVERAGE & $0.08(0.05)$ & $0.04(0.03)$ & $0.06(0.04)$ \\
\hline \multirow{6}{*}{$\begin{array}{c}\text { Maximum Slope } \\
\text { (Automatic Young's) } \\
{[\mathrm{mm} / \mathrm{mm}]}\end{array}$} & FARO 44 & $1.51(0.53)$ & $1.32(0.10)$ & $1.46(0.78)$ \\
\hline & FARO 52 & $1.42(1.09)$ & $1.56(0.72)$ & $1.48(1.11)$ \\
\hline & FARO 57 & $1.67(1.01)$ & $1.43(0.34)$ & $1.57(0.87)$ \\
\hline & FARO 60 & $1.54(0.65)$ & $1.23(0.32)$ & $1.41(1.05)$ \\
\hline & FARO 61 & $1.34(0.09)$ & $1.45(0.09)$ & $1.38(0.56)$ \\
\hline & AVERAGE & $1.50(0.67)$ & $1.34(0.31)$ & $1.46(0.87)$ \\
\hline
\end{tabular}

N.B: Data in parenthesis represents the standard deviation.

Table 2. Mechanical Properties of NERICA varieties (Raw-Paddy) at $17 \%$ (d.b) under different loading positions.

\begin{tabular}{ccccc}
\hline \multirow{3}{*}{ Mechanical Properties } & & \multicolumn{3}{c}{ Loading Positions } \\
\cline { 3 - 5 } & NERICA Variety & Major Diameter & Minor Diameter & $\begin{array}{c}\text { Intermediate } \\
\text { Diameter }\end{array}$ \\
\hline & FARO 44 & $212.45(2.13)$ & $8.47(0.78)$ & $87.64(1.67)$ \\
& FARO 52 & $214.15(1.88)$ & $5.72(0.55)$ & $73.29(2.08)$ \\
Maximum Load at Rupture [N] & FARO 57 & $215.66(1.76)$ & $15.51(1.02)$ & $79.07(0.99)$ \\
& FARO 60 & $240.48(2.09)$ & $11.72(0.89)$ & $84.78(1.21)$ \\
& FARO 61 & $260.83(1.86)$ & $11.50(0.67)$ & $96.78(0.77)$ \\
& AVERAGE & $228.71(1.94)$ & $10.58(0.78)$ & $84.31(1.34)$ \\
\hline & FARO 44 & $1.86(0.98)$ & $1.19(0.99)$ & $1.63(0.64)$ \\
Compressive Extension at & FARO 52 & $1.58(0.66)$ & $1.69(0.89)$ & $1.74(0.32)$ \\
Maximum Load [mm & FARO 57 & $1.96(0.95)$ & $2.19(0.11)$ & $1.99(0.31)$ \\
& FARO 60 & $1.60(0.56)$ & $1.61(0.34)$ & $1.62(0.64)$ \\
& FARO 61 & $1.11(0.08)$ & $1.49(0.57)$ & $1.38(0.44)$ \\
& AVERAGE & $1.62(0.65)$ & $1.64(0.58)$ & $1.67(0.47)$ \\
\hline & FARO 44 & $180.78(1.87)$ & $7.96(0.57)$ & $55.34(0.89)$ \\
Load at Bioyield Point [N] & FARO 52 & $177.45(1.07)$ & $4.67(0.45)$ & $46.76(1.08)$ \\
& FARO 57 & $181.93(1.11)$ & $11.17(1.02)$ & $51.39(0.64)$ \\
& FARO 60 & $110.42(0.87)$ & $8.12(0.88)$ & $66.17(2.08)$ \\
& FARO 61 & $215.54(0.99)$ & $7.89(0.94)$ & $73.35(1.99)$ \\
& AVERAGE & $173.22(1.18)$ & $7.96(0.77)$ & $58.61(1.34)$ \\
\hline Toughness & FARO 44 & $10.89(0.75)$ & $8.34(0.09)$ & $9.27(0.67)$ \\
(N/mm $\left.{ }^{2}\right)$ & FARO 52 & $9.01(0.08)$ & $6.78(0.53)$ & $8.01(0.99)$
\end{tabular}




\begin{tabular}{|c|c|c|c|c|}
\hline & FARO 57 & $8.06(0.23)$ & $7.29(0.88)$ & $7.67(0.46)$ \\
\hline & FARO 60 & $8.32(0.89)$ & $4.32(0.42)$ & $5.89(0.57)$ \\
\hline & FARO 61 & $7.67(0.98)$ & $6.96(0.31)$ & $7.98(0.61)$ \\
\hline & AVERAGE & $8.79(0.59)$ & $6.74(0.22)$ & $7.76(0.66)$ \\
\hline \multirow{6}{*}{$\begin{array}{c}\text { Stiffness } \\
(\mathrm{N} / \mathrm{mm})\end{array}$} & FARO 44 & $39.32(1.36)$ & $36.73(1.77)$ & $37.93(0.09)$ \\
\hline & FARO 52 & $46.98(0.69)$ & $46.47(0.51)$ & $46.89(1.01)$ \\
\hline & FARO 57 & $54.56(0.89)$ & $48.87(2.01)$ & $50.43(1.77)$ \\
\hline & FARO 60 & $41.32(0.34)$ & $34.56(1.04)$ & $39.35(0.76)$ \\
\hline & FARO 61 & $45.21(0.21)$ & $41.97(0.44)$ & $43.78(0.78)$ \\
\hline & AVERAGE & $45.48(0.71)$ & $41.72(1.15)$ & $43.68(0.88)$ \\
\hline \multirow{6}{*}{$\begin{array}{l}\text { Compressive } \\
\text { Strength } \\
\left(\mathrm{N} / \mathrm{mm}^{2}\right)\end{array}$} & FARO 44 & $6.27(0.66)$ & $5.02(0.11)$ & $5.98(0.89)$ \\
\hline & FARO 52 & $5.02(0.41)$ & $4.56(0.17)$ & $4.89(0.65)$ \\
\hline & FARO 57 & $4.88(0.99)$ & $3.99(0.88)$ & $4.01(1.01)$ \\
\hline & FARO 60 & $5.89(0.57)$ & $4.11(0.43)$ & $5.12(0.88)$ \\
\hline & FARO 61 & $4.47(0.19)$ & $4.95(0.83)$ & $4.78(0.53)$ \\
\hline & AVERAGE & $5.31(0.56)$ & $4.53(0.48)$ & $4.96(0.79)$ \\
\hline \multirow{6}{*}{ Energy at Maximum Load [J] } & FARO 44 & $0.05(0.02)$ & $0.03(0.01)$ & $0.04(0.01)$ \\
\hline & FARO 52 & $0.06(0.02)$ & $0.04(0.01)$ & $0.05(0.02)$ \\
\hline & FARO 57 & $0.13(0.09)$ & $0.02(0.01)$ & $0.11(0.02)$ \\
\hline & FARO 60 & $0.06(0.03)$ & $0.03(0.02)$ & $0.05(0.01)$ \\
\hline & FARO 61 & $0.08(0.02)$ & $0.02(0.01)$ & $0.06(0.03)$ \\
\hline & $A V E R A G E$ & $0.08(0.04)$ & $0.03(0.01)$ & $0.06(0.02)$ \\
\hline \multirow{6}{*}{$\begin{array}{c}\text { Maximum Slope (Automatic } \\
\text { Young's) } \\
{[\mathrm{mm} / \mathrm{mm}]}\end{array}$} & FARO 44 & $1.56(0.87)$ & $1.56(0.09)$ & $1.56(0.14)$ \\
\hline & FARO 52 & $1.67(0.11)$ & $1.97(0.33)$ & $1.59(0.18)$ \\
\hline & FARO 57 & $1.98(0.99)$ & $1.46(0.13)$ & $1.93(1.01)$ \\
\hline & FARO 60 & $1.34(0.46)$ & $1.94(0.77)$ & $1.41(0.77)$ \\
\hline & FARO 61 & $1.72(0.44)$ & $1.48(0.97)$ & $1.86(0.93)$ \\
\hline & AVERAGE & $8.27(0.57)$ & $1.68(0.46)$ & $1.67(0.60)$ \\
\hline
\end{tabular}

N.B: Data in parenthesis represents the standard deviation.

Table 3. Mechanical Properties of NERICA varieties (Raw-Paddy) at $21.5 \%$ (d.b) under different loading positions.

\begin{tabular}{ccccc}
\hline \multirow{2}{*}{ Mechanical Properties } & \multirow{2}{*}{ NERICA Variety } & & Loading Positions \\
\cline { 3 - 5 } & FARO 44 & Major Diameter & Minor Diameter & Intermediate Diameter \\
\hline & FARO 52 & $128.25(2.19)$ & $6.12(1.02)$ & $54.67(0.99)$ \\
Maximum Load at & FARO 57 & $72.24(1.21)$ & $7.44(0.98)$ & $76.56(2.01)$ \\
Rupture [N] & FARO 60 & $168.14(2.08)$ & $10.92(0.45)$ & $51.05(0.98)$ \\
& FARO 61 & $50.92(0.99)$ & $5.92(0.09)$ & $30.34(0.93)$ \\
& AVERAGE & $103.49(1.51)$ & $7.25(0.75)$ & $61.97(1.36)$ \\
\hline & FARO 44 & $1.95(0.67)$ & $1.57(0.12)$ & $1.78(0.11)$ \\
& FARO 52 & $2.79(0.34)$ & $1.72(0.87)$ & $2.01(1.01)$ \\
Compressive Extension & FARO 57 & $1.98(0.98)$ & $2.52(0.11)$ & $1.91(0.06)$ \\
at Maximum Load [mm & FARO 60 & $1.63(0.09)$ & $1.95(0.91)$ & $1.79(0.78)$ \\
& FARO 61 & $1.95(0.08)$ & $1.36(0.57)$ & $1.56(0.65)$ \\
& AVERAGE & $2.06(0.43)$ & $1.83(0.52)$ & $1.81(0.52)$ \\
\hline & FARO 44 & $77.43(0.34)$ & $5.42(1.01)$ & $23.45(1.09)$ \\
Load at Bioyield Point & FARO 52 & $69.78(1.09)$ & $4.01(0.67)$ & $45.89(0.88)$ \\
[N] & FARO 57 & $53.23(0.89)$ & $5.81(0.88)$ & $33.78(0.76)$ \\
& FARO 60 & $115.3(2.04)$ & $7.12(0.98)$ & $56.76(1.11)$ \\
& FARO 61 & $23.13(0.55)$ & $4.73(0.31)$ & $21.67(0.79)$ \\
& AVERAGE & $67.77(0.98)$ & $5.42(0.77)$ & $36.31(0.93)$ \\
\hline & FARO 44 & $9.20(0.31)$ & $7.45(0.67)$ & $8.98(1.01)$ \\
Toughness & FARO 52 & $8.32(0.12)$ & $6.21(1.22)$ & $7.82(0.94)$ \\
(N/mm $\left.{ }^{2}\right)$ & FARO 57 & $7.68(0.33)$ & $6.11(0.34)$ & $7.01(0.89)$ \\
& FARO 60 & $7.04(0.11)$ & $4.08(0.21)$ & $5.87(1.01)$ \\
& FARO 61 & $6.78(0.13)$ & $5.96(0.76)$ & $6.23(0.49)$ \\
Stiffness & AVERAGE & $7.81(0.21)$ & $5.96(0.64)$ & $7.18(0.87)$ \\
(N/mm) & FARO 44 & $41.21(1.91)$ & $27.81(1.33)$ & $32.56(0.46)$ \\
& FARO 52 & $47.82(0.67)$ & $47.87(0.46)$ & $45.76(1.22)$ \\
& FARO 57 & $57.32(1.91)$ & $50.76(1.01)$ & $52.45(1.97)$ \\
& FARO 60 & $43.21(0.43)$ & $37.39(0.93)$ & $39.56(0.42)$ \\
& FARO 61 & $47.22(0.59)$ & $43.56(0.48)$ & $45.62(0.49)$
\end{tabular}




\begin{tabular}{ccccc} 
& AVERAGE & $47.36(1.10)$ & $41.48(0.84)$ & $43.19(0.91)$ \\
\hline & FARO 44 & $5.98(0.14)$ & $4.23(0.09)$ & $4.98(0.11)$ \\
Compressive & FARO 52 & $4.89(0.46)$ & $4.19(0.14)$ & $4.52(1.02)$ \\
Strength & FARO 57 & $4.06(0.89)$ & $3.22(0.34)$ & $3.78(0.89)$ \\
$\left(\right.$ N/mm $\left.{ }^{2}\right)$ & FARO 60 & $5.01(0.56)$ & $3.89(0.13)$ & $4.67(0.67)$ \\
& FARO 61 & $4.13(1.01)$ & $4.07(0.98)$ & $4.11(0.99)$ \\
& AVERAGE & $4.82(0.62)$ & $3.92(0.34)$ & $4.41(0.74)$ \\
\hline & FARO 44 & $0.11(0.04)$ & $0.04(0.01)$ & $0.09(0.02)$ \\
Energy at Maximum & FARO 52 & $0.11(0.04)$ & $0.03(0.01)$ & $0.06(0.03)$ \\
Load [J] & FARO 57 & $0.04(0.01)$ & $0.06(0.02)$ & $0.05(0.02)$ \\
& FARO 60 & $0.08(0.04)$ & $0.03(0.01)$ & $0.04(0.01)$ \\
& FARO 61 & $0.09(0.03)$ & $0.02(0.01)$ & $0.06(0.03)$ \\
& AVERAGE & $0.09(0.03)$ & $0.04(0.01)$ & $0.06(0.02)$ \\
\hline Maximum Slope & FARO 44 & $1.67(0.66)$ & $1.52(0.34)$ & $1.61(0.94)$ \\
(Automatic Young's) & FARO 52 & $1.34(0.19)$ & $1.61(0.08)$ & $1.49(0.99)$ \\
[mm/mm] & FARO 57 & $1.36(0.20)$ & $1.12(0.04)$ & $1.26(0.78)$ \\
& FARO 60 & $1.28(0.99)$ & $1.35(0.11)$ & $1.31(1.02)$ \\
& FARO 61 & $1.57(0.67)$ & $1.71(0.65)$ & $1.62(0.99)$ \\
& AVERAGE & $1.44(0.54)$ & $1.46(0.24)$ & $1.46(0.94)$ \\
\hline
\end{tabular}

N.B: Data in parenthesis represents the standard deviation.

Table 4. Mechanical Properties of NERICA varieties (Parboiled-Milled) at $12.5 \%$ (d.b) under different loading positions.

\begin{tabular}{|c|c|c|c|c|}
\hline \multirow[b]{2}{*}{ Mechanical Properties } & \multirow[b]{2}{*}{ NERICA Variety } & \multicolumn{3}{|c|}{ Loading Positions } \\
\hline & & Major Diameter & Minor Diameter & $\begin{array}{l}\text { Intermediate } \\
\text { Diameter }\end{array}$ \\
\hline \multirow{6}{*}{ Maximum Load at Rupture [N] } & FARO 44 & $103.61(2.05)$ & $87.72(1.09)$ & $92.76(1.23)$ \\
\hline & FARO 52 & $110.10(1.91)$ & $64.28(1.22)$ & $102.19(2.09)$ \\
\hline & FARO 57 & $122.87(0.76)$ & $94.90(0.99)$ & $112.89(2.11)$ \\
\hline & FARO 60 & $156.08(2.76)$ & $51.85(1.19)$ & $133.96(1.67)$ \\
\hline & FARO 61 & $102.90(1.95)$ & $84.62(1.96)$ & $95.76(0.89)$ \\
\hline & AVERAGE & $119.11(1.89)$ & $76.67(1.29)$ & $107.51(1.61)$ \\
\hline \multirow{6}{*}{$\begin{array}{l}\text { Compressive Extension at } \\
\text { Maximum Load [mm }\end{array}$} & FARO 44 & $0.66(0.12)$ & $1.78(0.89)$ & $0.76(0.17)$ \\
\hline & FARO 52 & $1.61(1.33)$ & $1.77(1.07)$ & $1.72(0.56)$ \\
\hline & FARO 57 & $1.08(0.54)$ & $1.49(0.41)$ & $1.25(0.43)$ \\
\hline & FARO 60 & $1.17(0.98)$ & $0.53(0.23)$ & $0.98(0.08)$ \\
\hline & FARO 61 & $0.89(0.34)$ & $2.06(0.34)$ & $1.76(0.56)$ \\
\hline & AVERAGE & $1.08(0.66)$ & $1.53(0.59)$ & $1.29(0.36)$ \\
\hline \multirow{6}{*}{ Load at Bioyield Point [N] } & FARO 44 & $63.76(0.99)$ & $61.01(1.09)$ & $45.56(2.91)$ \\
\hline & FARO 52 & $85.23(0.31)$ & $45.22(0.89)$ & $67.89(0.99)$ \\
\hline & FARO 57 & $83.56(0.96)$ & $81.41(2.11)$ & $82.67(1.22)$ \\
\hline & FARO 60 & $101.34(1.97)$ & $35.12(0.88)$ & $83.18(1.78)$ \\
\hline & FARO 61 & $68.37(1.01)$ & $59.21(0.32)$ & $67.78(0.49)$ \\
\hline & AVERAGE & $80.45(1.05)$ & $56.39(1.06)$ & $69.42(1.48)$ \\
\hline \multirow{6}{*}{$\begin{array}{l}\text { Toughness } \\
\left(\mathrm{N} / \mathrm{mm}^{2}\right)\end{array}$} & FARO 44 & $10.23(0.23)$ & $8.88(0.87)$ & $9.78(0.46)$ \\
\hline & FARO 52 & $10.04(0.56)$ & $8.07(1.01)$ & $9.09(1.01)$ \\
\hline & FARO 57 & $7.98(0.75)$ & $5.98(0.87)$ & $6.98(0.89)$ \\
\hline & FARO 60 & $8.42(0.99)$ & $6.21(1.01)$ & $7.23(1.03)$ \\
\hline & FARO 61 & $8.06(1.01)$ & $6.77(0.99)$ & $7.11(0.87)$ \\
\hline & AVERAGE & $8.946(0.71)$ & $7.18(0.95)$ & $8.04(0.85)$ \\
\hline \multirow{6}{*}{$\begin{array}{l}\text { Stiffness } \\
(\mathrm{N} / \mathrm{mm})\end{array}$} & FARO 44 & $34.99(1.52)$ & $31.88(0.78)$ & $32.67(1.34)$ \\
\hline & FARO 52 & $37.81(0.97)$ & $43.11(0.87)$ & $41.46(0.84)$ \\
\hline & FARO 57 & $45.87(0.47)$ & $44.75(1.45)$ & $44.21(1.78)$ \\
\hline & FARO 60 & $37.01(1.01)$ & $32.04(0.67)$ & $34.12(2.02)$ \\
\hline & FARO 61 & $40.22(0.91)$ & $38.22(0.33)$ & $39.01(0.69)$ \\
\hline & AVERAGE & $39.18(0.98)$ & $38.01(0.82)$ & $38.29(1.33)$ \\
\hline \multirow{6}{*}{$\begin{array}{c}\text { Compressive } \\
\text { Strength } \\
\left(\mathrm{N} / \mathrm{mm}^{2}\right)\end{array}$} & FARO 44 & $6.44(1.01)$ & $5.13(0.34)$ & $5.98(0.09)$ \\
\hline & FARO 52 & $4.99(0.97)$ & $4.98(0.11)$ & $4.67(0.57)$ \\
\hline & FARO 57 & $5.11(0.67)$ & $4.49(0.34)$ & $5.56(0.18)$ \\
\hline & FARO 60 & $5.73(0.89)$ & $4.16(0.88)$ & $5.15(1.71)$ \\
\hline & FARO 61 & $4.97(1.03)$ & $4.44(1.06)$ & $4.19(0.65)$ \\
\hline & AVERAGE & $5.45(0.92)$ & $4.64(0.55)$ & $5.11(0.64)$ \\
\hline
\end{tabular}




\begin{tabular}{ccccc}
\hline & FARO 44 & $0.09(0.02)$ & $0.08(0.02)$ & $0.08(0.03)$ \\
& FARO 52 & $0.08(0.03)$ & $0.03(0.03)$ & $0.07(0.04)$ \\
Energy at Maximum Load [J] & FARO 57 & $0.11(0.02)$ & $0.04(0.01)$ & $0.10(0.06)$ \\
& FARO 60 & $0.04(0.01)$ & $0.02(0.01)$ & $0.03(0.01)$ \\
& FARO 61 & $0.06(0.03)$ & $0.08(0.04)$ & $0.07(0.04)$ \\
& AVERAGE & $0.08(0.02)$ & $0.05(0.02)$ & $0.07(0.04)$ \\
\hline & FARO 44 & $1.46(0.99)$ & $1.43(0.43)$ & $1.37(0.45)$ \\
Maximum Slope (Automatic & FARO 52 & $1.53(0.21)$ & $1.25(0.91)$ & $1.49(0.87)$ \\
Young's) & FARO 57 & $1.23(0.67)$ & $1.43(0.67)$ & $1.32(0.57)$ \\
[mm/mm] & FARO 60 & $1.53(0.09)$ & $1.36(0.45)$ & $1.51(0.89)$ \\
& FARO 61 & $1.75(0.69)$ & $1.31(0.56)$ & $1.69(0.49)$ \\
& AVERAGE & $1.50(0.53)$ & $1.36(0.61)$ & $1.48(0.65)$ \\
\hline
\end{tabular}

N.B: Data in parenthesis represents the standard deviation.

Table 5. Mechanical Properties of NERICA varieties (Parboiled-Milled) at 17\% (d.b) under different loading positions.

\begin{tabular}{|c|c|c|c|c|}
\hline \multirow[b]{2}{*}{ Mechanical Properties } & \multirow[b]{2}{*}{ NERICA Variety } & \multicolumn{3}{|c|}{ Loading Positions } \\
\hline & & Major Diameter & Minor Diameter & $\begin{array}{l}\text { Intermediate } \\
\text { Diameter }\end{array}$ \\
\hline \multirow{6}{*}{ Maximum Load at Rupture [N] } & FARO 44 & $105.49(2.31)$ & $84.90(1.94)$ & $89.19(0.56)$ \\
\hline & FARO 52 & $111.10(2.45)$ & $28.41(0.54)$ & $93.76(1.45)$ \\
\hline & FARO 57 & $74.94(1.88)$ & $26.92(1.02)$ & $80.93(2.71)$ \\
\hline & FARO 60 & $128.87(2.09)$ & $71.78(1.11)$ & $110.33(2.33)$ \\
\hline & FARO 61 & $110.99(1.88)$ & $71.14(0.66)$ & $94.07(0.94)$ \\
\hline & AVERAGE & $106.28(2.12)$ & $56.63(1.05)$ & $93.66(1.61)$ \\
\hline \multirow{6}{*}{$\begin{array}{l}\text { Compressive Extension at } \\
\text { Maximum Load [mm }\end{array}$} & FARO 44 & $3.21(0.78)$ & $1.60(0.98)$ & $2.45(0.67)$ \\
\hline & FARO 52 & $0.86(0.49)$ & $0.46(0.12)$ & $0.67(0.22)$ \\
\hline & FARO 57 & $0.97(0.98)$ & $0.90(0.65)$ & $0.91(0.43)$ \\
\hline & FARO 60 & $1.46(1.07)$ & $1.64(0.34)$ & $1.52(0.41)$ \\
\hline & FARO 61 & $1.44(0.66)$ & $2.67(1.01)$ & $1.98(0.85)$ \\
\hline & AVERAGE & $1.91(0.96)$ & $1.45(0.62)$ & $1.51(2.58)$ \\
\hline \multirow{6}{*}{ Load at Bioyield Point [N] } & FARO 44 & $81.56(0.95)$ & $52.67(2.05)$ & $51.89(1.55)$ \\
\hline & FARO 52 & $65.17(1.06)$ & $6.78(0.78)$ & $67.75(2.83)$ \\
\hline & FARO 57 & $46.12(0.76)$ & $10.21(0.55)$ & $48.65(1.08)$ \\
\hline & FARO 60 & $61.79(0.11)$ & $51.34(1.08)$ & $64.76(0.89)$ \\
\hline & FARO 61 & $65.76(1.15)$ & $48.46(0.89)$ & $62.56(0.67)$ \\
\hline & AVERAGE & $64.08(0.81)$ & $33.89(1.07)$ & $59.12(1.40)$ \\
\hline \multirow{6}{*}{$\begin{array}{l}\text { Toughness } \\
\left(\mathrm{N} / \mathrm{mm}^{2}\right)\end{array}$} & FARO 44 & $10.46(1.07)$ & $8.12(0.54)$ & $9.23(0.98)$ \\
\hline & FARO 52 & $8.89(0.08)$ & $6.32(0.11)$ & $7.98(1.09)$ \\
\hline & FARO 57 & $7.77(0.45)$ & $7.12(0.32)$ & $7.56(0.78)$ \\
\hline & FARO 60 & $6.75(0.34)$ & $4.01(0.08)$ & $5.21(0.71)$ \\
\hline & FARO 61 & $7.32(0.71)$ & $6.11(0.12)$ & $6.89(0.67)$ \\
\hline & AVERAGE & $8.24(0.53)$ & $20.94(0.23)$ & $7.38(0.85)$ \\
\hline \multirow{6}{*}{$\begin{array}{l}\text { Stiffness } \\
(\mathrm{N} / \mathrm{mm})\end{array}$} & FARO 44 & $36.49(1.12)$ & $32.12(0.99)$ & $35.17(2.01)$ \\
\hline & FARO 52 & $44.57(1.21)$ & $44.16(1.76)$ & $44.23(1.90)$ \\
\hline & FARO 57 & $51.39(0.31)$ & $47.81(1.87)$ & $48.91(1.46)$ \\
\hline & FARO 60 & $38.73(1.01)$ & $31.18(0.91)$ & $36.43(0.99)$ \\
\hline & FARO 61 & $44.15(0.07)$ & $38.79(0.69)$ & $41.12(0.78)$ \\
\hline & AVERAGE & $43.07(0.74)$ & $38.82(1.24)$ & $41.17(1.43)$ \\
\hline \multirow{6}{*}{$\begin{array}{c}\text { Compressive } \\
\text { Strength } \\
\left(\mathrm{N} / \mathrm{mm}^{2}\right)\end{array}$} & FARO 44 & $6.32(0.08)$ & $4.76(0.98)$ & $5.09(0.33)$ \\
\hline & FARO 52 & $4.99(0.42)$ & $4.03(1.01)$ & $4.52(0.78)$ \\
\hline & FARO 57 & $4.15(0.11)$ & $3.23(0.88)$ & $4.09(1.09)$ \\
\hline & FARO 60 & $5.33(0.43)$ & $3.72(0.98)$ & $4.98(0.98)$ \\
\hline & FARO 61 & $4.12(0.91)$ & $4.34(0.31)$ & $4.23(0.66)$ \\
\hline & AVERAGE & $4.98(0.39)$ & $4.12(0.83)$ & $4.58(0.77)$ \\
\hline \multirow{6}{*}{ Energy at Maximum Load [J] } & FARO 44 & $0.20(0.04)$ & $0.02(0.01)$ & $0.18(0.07)$ \\
\hline & FARO 52 & $0.11(0.13)$ & $0.04(0.02)$ & $0.08(0.05)$ \\
\hline & FARO 57 & $0.09(0.07)$ & $0.04(0.02)$ & $0.04(0.02)$ \\
\hline & FARO 60 & $0.07(0.02)$ & $0.05(0.03)$ & $0.06(0.01)$ \\
\hline & FARO 61 & $0.08(0.04)$ & $0.08(0.06)$ & $0.05(0.04)$ \\
\hline & AVERAGE & $0.11(0.13)$ & $0.05(0.14)$ & $0.08(0.04)$ \\
\hline
\end{tabular}




\begin{tabular}{ccccc}
\hline & FARO 44 & $1.65(0.23)$ & $1.54(0.03)$ & $1.55(0.19)$ \\
Maximum Slope (Automatic & FARO 52 & $1.45(0.11)$ & $1.57(0.88)$ & $1.48(0.98)$ \\
Young's) & FARO 57 & $1.72(0.07)$ & $1.45(0.93)$ & $1.61(0.87)$ \\
{$[\mathrm{mm} / \mathrm{mm}]$} & FARO 60 & $1.58(0.06)$ & $1.52(0.95)$ & $1.53(0.34)$ \\
& FARO 61 & $1.34(0.04)$ & $1.47(0.87)$ & $1.29(0.56)$ \\
& AVERAGE & $1.55(0.10)$ & $1.51(0.73)$ & $1.49(0.59)$ \\
\hline
\end{tabular}

N.B: Data in parenthesis represents the standard deviation.

Table 6: Mechanical Properties of NERICA varieties (Parboiled-Milled) at $21.5 \%$ (d.b) under different loading positions.

\begin{tabular}{|c|c|c|c|c|}
\hline \multirow[b]{2}{*}{ Mechanical Properties } & \multirow[b]{2}{*}{ NERICA Variety } & \multicolumn{3}{|c|}{ Loading Positions } \\
\hline & & $\begin{array}{c}\text { Major } \\
\text { Diameter }\end{array}$ & $\begin{array}{c}\text { Minor } \\
\text { Diameter }\end{array}$ & Intermediate Diameter \\
\hline \multirow{6}{*}{ Maximum Load at Rupture [N] } & FARO 44 & $44.94(2.09)$ & $38.88(2.08)$ & $40.78(2.07)$ \\
\hline & FARO 52 & $77.33(2.11)$ & $24.63(1.97)$ & $57.67(2.77)$ \\
\hline & FARO 57 & $78.16(1.88)$ & $22.05(1.77)$ & $61.13(1.09)$ \\
\hline & FARO 60 & $67.61(0.87)$ & $23.93(2.04)$ & $47.38(1.67)$ \\
\hline & FARO 61 & $49.74(0.67)$ & $34.29(1.77)$ & $38.89(0.87)$ \\
\hline & AVERAGE & $63.56(1.52)$ & $28.76(1.93)$ & $49.17(1.69)$ \\
\hline \multirow{6}{*}{$\begin{array}{c}\text { Compressive Extension at Maximum } \\
\text { Load }[\mathrm{mm}\end{array}$} & FARO 44 & $0.71(0.18)$ & $0.65(0.08)$ & $0.68(0.07)$ \\
\hline & FARO 52 & $0.87(0.05)$ & $0.50(0.02)$ & $0.57(0.12)$ \\
\hline & FARO 57 & $0.95(0.56)$ & $1.31(0.43)$ & $1.22(0.67)$ \\
\hline & FARO 60 & $0.59(0.12)$ & $1.38(0.54)$ & $1.27(0.68)$ \\
\hline & FARO 61 & $1.49(0.07)$ & $1.45(0.32)$ & $1.47(0.56)$ \\
\hline & AVERAGE & $0.92(0.21)$ & $1.06(0.28)$ & $1.04(0.62)$ \\
\hline \multirow{6}{*}{ Load at Bioyield Point [N] } & FARO 44 & $18.38(1.89)$ & $10.98(1.03)$ & $17.86(0.44)$ \\
\hline & FARO 52 & $26.01(1.01)$ & $14.51(1.08)$ & $23.21(1.22)$ \\
\hline & FARO 57 & $31.21(2.06)$ & $13.34(0.65)$ & $25.68(1.04)$ \\
\hline & FARO 60 & $25.27(0.87)$ & $10.21(0.67)$ & $18.56(0.67)$ \\
\hline & FARO 61 & $17.67(0.99)$ & $17.91(0.43)$ & $16.43(0.66)$ \\
\hline & AVERAGE & $23.71(1.36)$ & $13.39(0.77)$ & $20.35(0.81)$ \\
\hline \multirow{6}{*}{$\begin{array}{l}\text { Toughness } \\
\left(\mathrm{N} / \mathrm{mm}^{2}\right)\end{array}$} & FARO 44 & $8.98(0.99)$ & $7.11(0.11)$ & $8.21(0.88)$ \\
\hline & FARO 52 & $7.18(1.98)$ & $5.99(0.87)$ & $6.37(0.19)$ \\
\hline & FARO 57 & $7.32(0.89)$ & $5.87(0.56)$ & $6.89(0.98)$ \\
\hline & FARO 60 & $6.19(1.12)$ & $3.97(0.33)$ & $4.74(0.56)$ \\
\hline & FARO 61 & $6.55(1.87)$ & $5.24(0.67)$ & $6.01(0.17)$ \\
\hline & AVERAGE & $7.24(1.37)$ & $5.64(2.54)$ & $6.44(0.56)$ \\
\hline \multirow{6}{*}{$\begin{array}{l}\text { Stiffness } \\
(\mathrm{N} / \mathrm{mm})\end{array}$} & FARO 44 & $39.03(2.03)$ & $25.17(2.05)$ & $28.32(1.01)$ \\
\hline & FARO 52 & $45.11(2.92)$ & $45.22(1.07)$ & $45.15(2.07)$ \\
\hline & FARO 57 & $57.21(2.33)$ & $48.16(2.09)$ & $52.15(2.87)$ \\
\hline & FARO 60 & $40.14(1.44)$ & $35.22(1.99)$ & $38.76(0.87)$ \\
\hline & FARO 61 & $42.44(0.99)$ & $41.24(0.49)$ & $42.08(0.97)$ \\
\hline & AVERAGE & $44.79(1.94)$ & $39.00(1.54)$ & $41.29(1.56)$ \\
\hline \multirow{6}{*}{$\begin{array}{c}\text { Compressive } \\
\text { Strength } \\
\left(\mathrm{N} / \mathrm{mm}^{2}\right)\end{array}$} & FARO 44 & $5.32(0.98)$ & $4.04(0.92)$ & $5.12(0.06)$ \\
\hline & FARO 52 & $4.11(0.43)$ & $3.22(1.01)$ & $4.09(0.77)$ \\
\hline & FARO 57 & $3.98(0.87)$ & $2.98(0.88)$ & $3.41(0.42)$ \\
\hline & FARO 60 & $4.34(0.43)$ & $3.45(0.97)$ & $3.98(0.44)$ \\
\hline & FARO 61 & $3.78(0.09)$ & $3.99(0.67)$ & $3.62(0.76)$ \\
\hline & AVERAGE & $4.31(0.56)$ & $3.54(0.98)$ & $4.04(0.49)$ \\
\hline \multirow{6}{*}{ Energy at Maximum Load [J] } & FARO 44 & $0.09(0.02)$ & $0.08(0.02)$ & $0.08(0.02)$ \\
\hline & FARO 52 & $0.11(0.05)$ & $0.04(0.01)$ & $0.10(0.03)$ \\
\hline & FARO 57 & $0.09(0.04)$ & $0.05(0.02)$ & $0.06(0.01)$ \\
\hline & FARO 60 & $0.08(0.67)$ & $0.03(0.01)$ & $0.07(0.02)$ \\
\hline & FARO 61 & $0.08(0.03)$ & $0.02(0.01)$ & $0.06(0.02)$ \\
\hline & AVERAGE & $0.09(0.16)$ & $0.04(0.01)$ & $0.07(1.00)$ \\
\hline \multirow{6}{*}{$\begin{array}{c}\text { Maximum Slope (Automatic Young's) } \\
{[\mathrm{mm} / \mathrm{mm}]}\end{array}$} & FARO 44 & $1.46(0.98)$ & $1.34(0.34)$ & $1.39(0.54)$ \\
\hline & FARO 52 & $1.64(0.86)$ & $1.54(0.67)$ & $1.45(0.67)$ \\
\hline & FARO 57 & $1.69(0.67)$ & $1.52(0.55)$ & $1.61(0.09)$ \\
\hline & FARO 60 & $1.23(0.17)$ & $1.19(0.07)$ & $1.21(0.56)$ \\
\hline & FARO 61 & $1.56(0.53)$ & $1.27(0.32)$ & $1.44(0.87)$ \\
\hline & AVERAGE & $7.58(0.64)$ & $1.37(0.39)$ & $1.42(0.55)$ \\
\hline
\end{tabular}

N.B: Data in parenthesis represents the standard deviation. 
The values of the mechanical properties of the grain were found to be a function of moisture content $(12.5$ to $21.5 \%)(\mathrm{db})$. The relationship between the moisture content and the mechanical properties was statistically significant $(\mathrm{p}<0.05)$. The maximum load at rupture decreased with an increase in moisture content. The results ranged from 363.73 to $50.92 \mathrm{~N}, 125.35$ to $51.05 \mathrm{~N}$, and 18.46 to $5.44 \mathrm{~N}$, at major, intermediate, and minor diameters loading positions respectively for NERICA Raw-Paddy.

And for NERICA Parboiled-Milled, the maximum load at rupture also ranged from 156.08 to $44.94 \mathrm{~N}, 133.96$ to $38.89 \mathrm{~N}$, and 94.90 to $22.05 \mathrm{~N}$, at major, intermediate, and minor diameters loading positions respectively with an increase in moisture content, Tables 1 to 6 . Rupture force is the total or maximum load required for an agricultural material to break or be deformed, [14]. Also, from Tables 1 to 6, compressive extension at maximum load (deformation) ranged from 0.50 to $2.79 \mathrm{~mm}, 0.68$ to $2.01 \mathrm{~mm}$, and 0.92 to $2.52 \mathrm{~mm}$, at major, intermediate, and minor diameters loading positions respectively, with an increase in moisture content (12.5 to $21.5 \%$ ) for NERICA RawPaddy, and 0.59 to $1.61 \mathrm{~mm}, 0.57$ to $2.45 \mathrm{~mm}$, and 0.46 to $2.67 \mathrm{~mm}$, at major, intermediate, and minor diameters loading positions respectively, with an increase in moisture content (12.5 to $21.5 \%$ ) (db) for NERICA Parboiled-Milled. Load at bioyield point decreased as moisture content increases. Load at bioyield point is described as that point at which a rise in deformation is marked with a change of force in some agricultural products. Results indicated that load at bioyield point ranged from 321.56 to $23.13 \mathrm{~N}, 91.45 \mathrm{~N}$ to $21.67 \mathrm{~N}$, and 11.17 to $4.01 \mathrm{~N}$, at major, intermediate, and minor diameters loading positions respectively for NERICA Raw-Paddy. And for NERICA Parboiled-Milled, load at bioyield point ranged from 101.34 to $17.67 \mathrm{~N}, 83.18$ to 16.43 $\mathrm{N}$, and 81.41 to $6.78 \mathrm{~N}$, at major, intermediate, and minor diameters loading positions respectively. Toughness also decreased with an increase in moisture content. Toughness is the ability of a material to absorb energy (or withstand shock) and plastically deform without fracturing (or rupturing), [14]. The result ranged from 11.58 to $6.78 \mathrm{~N} / \mathrm{mm}^{2}$, 10.12 to $5.87 \mathrm{~N} / \mathrm{mm}^{2}$, and 9.01 to $4.08 \mathrm{~N} / \mathrm{mm}^{2}$, at major, intermediate and minor diameters loading positions for NERICA Raw-Paddy, and 10.46 to $6.19 \mathrm{~N} / \mathrm{mm}^{2}, 9.78$ to $6.01 \mathrm{~N} / \mathrm{mm}^{2}$, and 8.12 to $4.01 \mathrm{~N} / \mathrm{mm}^{2}$ at major, intermediate, and minor diameters loading positions for NERICA Parboiled-Milled. Stiffness was the ability of NERICA varieties to resist deformation in response to the applied force. Increase in moisture content resulted to an increase in stiffness. The result ranged from 36.89 to $57.32 \mathrm{~N} / \mathrm{mm}$, 32.56 to $52.45 \mathrm{~N} / \mathrm{mm}$, and 27.81 to $50.76 \mathrm{~N} / \mathrm{mm}$ at major, intermediate, and minor diameters loading positions respectively for NERICA Raw-Paddy. For NERICA Parboiled-Milled, stiffness ranged from 34.99 to $57.21 \mathrm{~N} / \mathrm{mm}, 28.32$ to $52.15 \mathrm{~N} / \mathrm{mm}$, and 25.17 to $48.16 \mathrm{~N} / \mathrm{mm}$ at major, intermediate, and minor diameters loading positions respectively. The compressive strength of NERICA, which is the capacity of the material to withstand loads tending to reduce size, decreased with an increase in moisture content. The result obtained ranged from 6.91 to $4.06 \mathrm{~N} / \mathrm{mm}^{2}, 6.01$ to $3.78 \mathrm{~N} / \mathrm{mm}^{2}$, and 5.21 to $3.22 \mathrm{~N} / \mathrm{mm}^{2}$ at major, intermediate, and minor diameters loading positions respectively for NERICA Raw-Paddy, and from 6.44 to $3.78 \mathrm{~N} / \mathrm{mm}^{2}, 5.98$ to $3.41 \mathrm{~N} / \mathrm{mm}^{2}$, and 5.13 to $2.98 \mathrm{~N} / \mathrm{mm}^{2}$ at major, intermediate, and minor diameters loading positions respectively for NERICA Parboiled-Milled. Energy at maximum load for NERICA varieties decreased as moisture content increased. The result ranged from 0.14 to $002 \mathrm{~J}$. 
This is the amount of energy NERICA varieties can absorb at maximum load for both major, minor and intermediate diameters loading positions as moisture content increases from 12.5 to $21.5 \%$ (db).

Statistical analysis was carried out to study the effects of moisture content on the mechanical properties of NERICA varieties. Individual regressions, regressing mechanical properties with moisture contents were carried out on each variety. The values of the mechanical properties of the NERICA samples were found to be a function of moisture content.

All the mechanical properties showed similar pattern with increase in moisture content and decrease in load, at both major diameter, minor diameter and intermediate diameter loading positions, except the compressive extension at maximum load and the stiffness that increased with an increase in moisture content. This is as a result of the soft texture of the seeds at high moisture content levels. Similar relationships between moisture content and some mechanical properties, though, under horizontal and vertical loading orientations were reported by [7], for some NERICA varieties; [15], for locust bean; [10], for bush mango; [9], for mucuna flagellipes nut; [16], for brown rice; and [17] for Soybean grains.

\section{CONCLUSION}

The study proved the significance of the effect of moisture content of the mechanical properties of NERICA, and established the negligibility of variety factors and effects in the engineering processing of NERICA. There were significant effects of moisture content $(\mathrm{p}<0.05)$ on all parameters studied. Results of the mechanical properties of NERICA generated will be highly needed in the design of dehullers, threshers and mills, and destoning machines for NERICA, especially in the determination of the power requirement of the equipment, and also, for the design of silos for storing of NERICA. These results obtained will serve as useful guides to farmers and processors of agro-products for proper harvesting and processing operations, such as size reduction or milling of the crop, and also assist in the design of wide range of handling and processing equipment of NERICA, such as transport facilities, storage facilities, cleaning, grading, and sizing equipment.

\section{REFERENCES}

[1] WARDA, (2008). West Africa Rice Center/FAO/SAA issues on NERICA; the New Rice for Africa, a Compendium. E.A. Samado, R.G. Guei and S.O. Keya (eds). Cotonu, Benin. Africa Rice Centre (WARDA); Rome, Italy; FAO; Tokyo, Japan: Sasakawa Africa Association; pp: 210.

[2] Udemezue, J.C. (2018). Analysis of Rice and Consumption Trends in Nigeria. J. P. Sci. Crop Protection, 1(3); pp: 305.

[3] Eze, P.C. and Oluka, S.I. (2014). Selected Physical and Aerodynamics Properties of NERICA (New Rice for Africa). Journal of Agricultural Engineering and Technology (JAET), 22 (3); pp 47- 60.

[4] Mehdi, G.V. Hossein, M., Ali, J., Shahin, R., Mohsen, H., and Kamran, K. (2007). Some Engineering Properties of Paddy (var. Sazandegi). Intl. Journal of Agric \& Biology, 9 (5); pp: 763 - 766 . 
[5] Mohapatra, D. and Bal, S. (2004). Wear of Rice in an abrasive Milling Operation, Part 2: Prediction of bulk Temperature rice. Bio-systems Engr., 89; pp: $101-108$.

[6] Sunmonu, M.O., Iyanda, M.O., Odewole, M.M., and Moshood, A.N. (2015). Determination of Some Mechanical Properties of Almond Seed Related to Design of Food Processing Machines. Nigerian Journal of Technological Dev., 12 (1); pp: 22-26.

[7] Agu, O.S. and Oluka, S.I. (2013). Selected Physical and Mechanical Properties of Some NERICA paddy. Journal of Experimental Research, 1(1); pp: 27-40.

[8] Gayin, J., Manful, J.T. and Johnson, P-N.T. (2009). Rheological and Sensory Properties of Rice varieties from Improvement Programmes in Ghana. International Food Research Journal, 16; pp: 167-174.

[9] Aviara, N.A., Onuh, O.A., and Ehiabhi, S.E. (2012). Influence of Moisture Content and Loading orientation on Some Mechanical Properties of Mucuna flagellipes nut. Res. Agr. Eng, 58; pp: 66-72.

[10] Nwigbo, S.C., Ngini, J.O., and Atuanya, C.U. (2013). Physical and Mechanical Properties of Irvingia Gabonesis and Irvingia Wombolu at varying Moisture Content and Temperature. International J. of Multidisciplinary Sciences and Eng., 4(6); pp: 10-14.

[11] Ituen, E.U. and Ukpakha, A.C. (2011). Improved Method of Par-boiling Paddy for a better-quality Rice. World J.of App. Science and Technology, Vol.3. No.1; Pp: 31 - 40.

[12] Nalladullai, K., Alagusundaram, K., and Gayathri, P. (2002). Airflow Resistance of Paddy and its byproducts. Bio-systems Engr., 83; pp: $67-75$.

[13] Mohsenin, N. (1986). Physical Properties of Plant and Animal Materials, second edition, Gordon and Breach, New York, USA.

[14] Stroshine, R.L. (2004). Physical Properties of Agricultural Materials and Food Products Purdue University Publishers, Indiana, USA.

[15] Sadiku, A.O. and Bamgboye, I. (2014). Moisture dependent mechanical and thermal properties of Locust bean (Parkia biglobosa). Agric Eng Int: CIGR Journal 16 (1); pp: 99 $-106$.

[16] Cao, W., Nishiyama, Y., and Koide, S. (2004). Physicochemical, Mechanical and Thermal properties of brown rice grain with various moisture contents. International Journal of Food Science and Technology, 39; pp: 899-906.

[17] Tavakoli, H., Rajabipour, A. and Mohtasebi, S.S. (2009). Moisture dependent Engineering properties of soybean grains. Agricultural Engineering International: the CIGR e-journal, 11; pp: $1-10$.

\section{UTICAJ SADRŽAJA VLAGE I POZICIJE ZA UTOVAR NA MEHANIČKE OSOBINE NOVE SORTE (NERICA) PIRINČA ZA AFRIKU}

Eze Paul Chukwuka ${ }^{* 1}$, Oluka Sylvester Ikechuwu ${ }^{1}$, and Eze Chikaodili Nkechi ${ }^{1}$

${ }^{1}$ Agricultural and Bioresource Engineering,

Enugu State University of Science and Technology,

Enugu, Nigeria.

\footnotetext{
*Corresponding Author. E-mail: paul.eze@esut.edu.ng
} 
Sažetak: Mehaničke osobine nove sorte pirinča za Afriku (NERICA) određene su kod različitih sadržaja vlage od 12,5, 17 i 21,5 \% (db), i položaja punjenja glavnih, malih i srednjih prečnika otvora test uređaja Instron Universal Testing Machine (UTM). Ispitane mehaničke osobine zrna su: vrednost maksimalnog opterećenja i oštećenja kod pucanja i smicanja; vrednosti ekstenzije (istezanja) i pritiska zrna kao biološkog materijala; najveća energija opterećenja zrna, krutost i najveće klizanje zrna na nagibu. Dobijeni rezultati pokazuju da opterećenje i sadržaj vlage utiču na ispitivana mehanička svojstva determinisana sa $(\mathrm{p}<0,05)$.

Rezultati ispitivanja su pokazali da je najveće opterećenje od 363,73 N do $50,92 \mathrm{~N} \mathrm{i}$ oštećenje kod pucanja, kompresija u rasponu $0,50 \mathrm{~mm}$ do $2,79 \mathrm{~mm}$; a opterećenje na preseku biološke izdržljivosti od 11,58 do $6,78 \mathrm{~N} / \mathrm{mm}^{2}$; respektivno za opterećenje najvećeg prečnika zrna NERICA Rav-Paddi; od 18.46 do $5.44 \mathrm{~N}$; 0,92 do 2,52 mm; 9.01 do $4.08 \mathrm{~N} / \mathrm{mm}^{2}$ za NERICA Rav-Paddi opterećenje manjeg prečnika zrna.

Za NERICA Parboiled-Milled zrno pirinča rezultati na pozicijama punjenja glavnog prečnika i malog prečnika test uređaja su raspoređeni na sledeći način: maksimalno opterećenje pri pucanju (156,08 do 44,94 N; 94,90 do 22,05 N); kompresija zrna $(0,59$ do $1,61 \mathrm{~mm} ; 0,46$ do $2,67 \mathrm{~mm}$ ). Regresijska analiza mehaničkih svojstava i sadržaja vlage u zrnu pokazuje pozitivna korelacija između ispitivanih parametara. Bilo je značajnih efekata uticaja sadržaja vlage (pozitivna korelacija $p<0,05$ ) na sve ispitivane parametre.

Ključne reči: Sadržaj vlage, mehaničke osobine, vrste/pozicija opterećenja, NERICA.

Prijavljen:

Submitted:

14.09.2019.

Ispravljen:

Revised:

13.02.2020.

Prihvaćen:

Accepted:

21.02.2020. 